\title{
Articles
}

\section{DYNAMIC ANALYSIS OF SELECTED EUROPEAN STOCK MARKETS}

\author{
Jiří Trešl, Dagmar Blatná*
}

\begin{abstract}
:
The behaviour of selected European stock indices in the period 2001-2005 was analysed. UKX (GB), DAX (Germany), CAC (France) and MIBTEL (Italy) represented well established West European markets, whereas PX-50 (Czech Republic), SKSM (Slovak Republic), BUX (Hungary) and WIG (Poland) were the examples of Central European emerging ones. The subject of this analysis were logarithmic daily returns computed from closing values of corresponding indices. Cross correlation function reached typical values 0.7 (West Europe) and 0.4 (Central Europe) excepting the Slovak Republic. The patterns of both common and solitary movements were revealed with the use of principal component and cluster analysis. To establish some dynamical relations in return time-series, vector autoregression models and Granger causality tests were employed. As for West Europe, the causal chain UKX_MIBTEL_DAX_CAC was revealed. On the other hand, the form of this chain for Central Europe was PX-50_BUX_WIG. Finally, the behaviour of both BUX and WIG returns was strongly determined by all West European counterparts.
\end{abstract}

Keywords: stock indices, return modelling, financial time series, Granger causality

JEL Classification: C32, G15

\section{Introduction}

During the last two decades, derivatives have become increasingly important in the world of finance. Futures and options are now traded actively on many exchanges throughout the world. Many different types of forward contracts, swaps, options and other derivatives are regularly traded by financial institutions and funds. However, it is important to realize, a derivative can be defined as a financial instrument whose value depends on the values of other, more basic, underlying variables (Hull, 2006). For

* University of Economics, Faculty of Informatics and Statistics, Prague (tres1@vse.cz, blatna@vse.cz).

Financial support from the GACR (Project No. 402/05/0128) is strongly appreciated. 
example, a stock option is a derivative, whose value is dependent on the stock price. Therefore, the analysis of stock prices and stock returns can be highly useful in problems of portfolio selection (Sharpe and Alexander, 1990). This problem has long history starting from pioneering work (Markowitz, 1952) towards to the creation of CAPM model (Sharpe, 1964), (Mossin, 1966), (Fama, 1968).

An important role in understanding the changing character of capital markets can be ascribed to Bruno Solnik, who published an article Why not diversify internationally rather than domestically? in 1974. He analysed here the rapid pace of international investing due to a change in mentality with respect to many factors. First, the benefits of international diversification in terms of risk and return have progressively been recognized. A second factor is the deregulation and internationalization of financial markets throughout the world. This integration of financial markets leads to reduced costs, easier access to information and the development of a worldwide expertise by major financial institutions (Solnik, 1996).

Financial time series analysis is one of the most rapidly developing branches at the boundary of statistics and econometrics. However, there is a key feature that distinguishes financial time series analysis from "usual" time series analysis. Both financial theory and its empirical time series contain always an element of uncertainty. For example, there are various definitions of asset volatility and this quantity is unobservable for stock return series. Thus, due to uncertainty added, statistical theory and methods play an important role (Tsay, 2002).

\section{Input Data and Descriptive Methods}

The main aim of this study is to compare the behaviour of stock markets both in West Europe and in Central-European emerging markets. Input data are given as daily values of corresponding stock indices $x_{t}$ during the period 1998-2004, i.e. 1655 daily values. As for indices, we have selected $U K X$ (GB), DAX (Germany), CAC (France) and MIBTEL (Italy) and, on the other hand, PX-50 (Czech Republic), SKSM (Slovak Republic), WIG (Poland) and $B U X$ (Hungary). The subjects of our analysis were logarithmic returns (further only returns) expressed as percentage and computed as

$$
y_{t}=100\left(\ln x_{t}-\ln x_{t-1}\right)
$$

This is a common practice in financial time series analysis, because continuously compounded multiperiod return is simply the sum of continuously compounded one-period returns involved (Tsay, 2002).

First, some elementary summary statistics related to daily returns were computed. The results obtained are compiled in the Table 1.

Clearly, there are no distinct differences in unconditional variability, i.e. standard deviation and mean absolute deviation. Further, extreme values for BUX and WIG are roughly twice the size of ones for PX-50 and UKX. Notice that the kurtosis is always positive, i.e. the empirical distribution exhibits greater peakedness in comparison with normal one. This tendency to more pronounced peaks and heavy tails seems to be a persistent feature in financial time series (Tsay, 2002). 
Table 1

Summary Statistics for Returns

\begin{tabular}{|l|r|r|r|r|r|r|r|r|}
\hline & \multicolumn{1}{|c|}{ BUX } & PX50 & SKSM & WIG & CAC & DAX & MIBTEL & UKX \\
\hline Median & 0.05 & 0.03 & 0.00 & 0.04 & 0.04 & 0.06 & 0.04 & 0.02 \\
\hline Standard deviation & 1.86 & 1.38 & 1.47 & 1.73 & 1.64 & 1.85 & 1.45 & 1.30 \\
\hline MAD & 0.89 & 0.78 & 0.61 & 0.84 & 0.91 & 1.03 & 0.73 & 0.70 \\
\hline Minimum & -15.53 & -7.08 & -11.48 & -13.54 & -7.02 & -8.73 & -10.47 & -5.59 \\
\hline Maximum & 16.32 & 6.22 & 5.96 & 13.53 & 13.97 & 15.27 & 14.43 & 6.82 \\
\hline Range & 31.85 & 13.30 & 17.44 & 27.07 & 20.99 & 24.00 & 24.90 & 12.41 \\
\hline Lower quartile & -0.86 & -0.73 & -0.54 & -0.85 & -0.89 & -1.04 & -0.68 & -0.70 \\
\hline Upper quartile & 0.93 & 0.83 & 0.65 & 0.86 & 0.91 & 1.05 & 0.77 & 0.72 \\
\hline Interquartile range & 1.79 & 1.56 & 1.19 & 1.71 & 1.8 & 2.09 & 1.45 & 1.42 \\
\hline Skewness & -0.29 & -0.04 & -0.67 & 0.36 & 0.30 & 0.20 & 0.18 & -0.01 \\
\hline Kurtosis & 12.87 & 1.90 & 6.30 & 9.99 & 4.61 & 4.08 & 9.17 & 2.01 \\
\hline
\end{tabular}

Figure 1

Time Series of PX-50 Daily Values

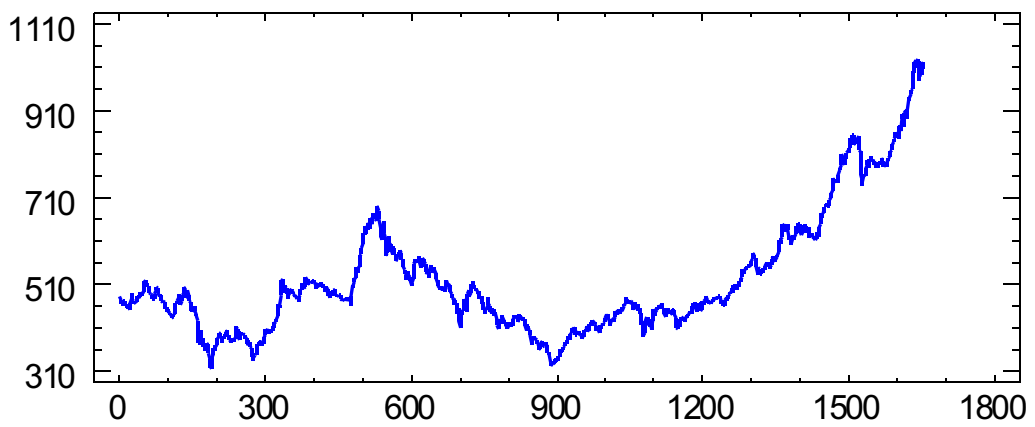

Figure 2

Time Series of PX-50 Daily Returns (in \%)

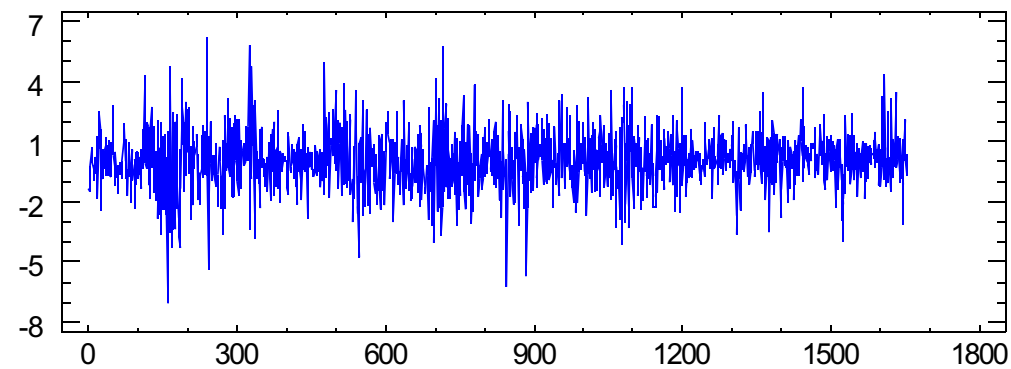

As for a suitable theoretical model for returns distribution fitting, the following types were examined: Laplace, logistic, normal and Student's t distributions (Evans et al.1993, Johnson et al. 1995). The results obtained are summarised in Table 2. 
Table 2

P-values for Distribution Fitting - Chi-squared Test

\begin{tabular}{|l|c|c|c|c|c|c|c|c|}
\hline & BUX & PX50 & SKSM & WIG & CAC & DAX & MIBTEL & UKX \\
\hline Laplace & 0.079 & 0.005 & 0.000 & $\mathbf{0 . 3 0 2}$ & 0.000 & 0.000 & 0.012 & 0.007 \\
\hline Logistic & $\mathbf{0 . 4 4 6}$ & 0.175 & 0.000 & 0.002 & $\mathbf{0 . 0 1 4}$ & $\mathbf{0 . 0 6 7}$ & 0.000 & 0.170 \\
\hline Normal & 0.000 & 0.000 & 0.000 & 0.000 & 0.000 & 0.000 & 0.000 & 0.000 \\
\hline Student's t & 0.356 & $\mathbf{0 . 2 8 0}$ & 0.000 & 0.014 & 0.000 & 0.000 & $\mathbf{0 . 0 2 7}$ & $\mathbf{0 . 4 3 3}$ \\
\hline
\end{tabular}

Figure 3

Distribution Fitting: PX-50 Returns versus t-Distribution

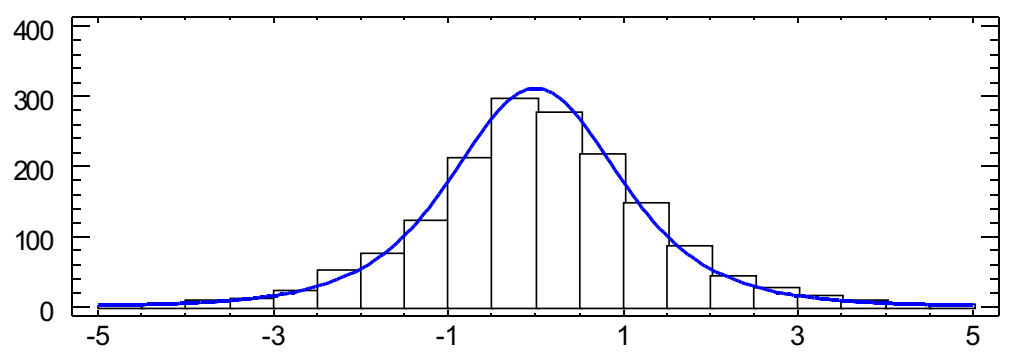

Thus, logistic and Student's t distributions proved to be the best ones, whereas normal distribution failed in all cases, because it has systematically lower kurtosis.

To reveal possible relations among individual returns, correlations were computed with the following results:

Table 3

Correlations among Returns (all values are statistically significant at $5 \%$ level)

\begin{tabular}{|l|c|c|c|c|c|c|c|c|}
\hline & BUX & PX50 & SKSM & WIG & CAC & DAX & MIBTEL & UKX \\
\hline BUX & XXXXX & 0.485 & - & 0.436 & 0.424 & 0.448 & 0.460 & 0.422 \\
\hline PX50 & & XXXXX & - & 0.378 & 0.416 & 0.406 & 0.399 & 0.392 \\
\hline SKSM & & & XXXXX & - & - & - & - & - \\
\hline WIG & & & & XXXXX & 0.306 & 0.318 & 0.310 & 0.305 \\
\hline CAC & & & & & XXXXX & 0.811 & 0.809 & 0.825 \\
\hline DAX & & & & & & XXXXX & 0.755 & 0.720 \\
\hline MIBTEL & & & & & & & XXXXX & 0.724 \\
\hline UKX & & & & & & & & XXXXX \\
\hline
\end{tabular}

Thus, one can state, the highest correlations are among West-European indices: CAC/UKX (0.82), CAC/DAX (0.81) and CAC/MIBTEL (0.81). On the other hand, as for Central-European indices, the correlations are weaker and the highest ones among BUX/PX-50 (0.48), BUX/WIG (0.44) and WIG/PX-50 (0.38). Moreover, the returns of SKSM exhibit some kind of "solitaire" behaviour, being completely uncorrelated. The same conclusions were drawn with the use of both principal component and cluster analysis (see Chapter 3). 


\section{Modelling}

The next step is the possibility of modelling the return time series. First, the behaviour of autocorrelation function (ACF) was investigated. In general, ACF values statistically significant at $5 \%$ level and varying between -0.113 (UKX) and +0.119 (WIG) occured up to relatively high orders. First-order positive ACF values were revealed in three cases (BUX, PX50, WIG); on the other hand, their counterparts for West-European returns were missing.

On the other hand, ACF computed for absolute values of returns reach typical values 0.15 and decrease very slowly, so that all values up to lag $=20$ are still statistically significant at 5\% level. This is a crucial difference in comparison with ACF of white noise process. For this reason, we used combined AR-GARCH models suitable for the modelling in the presence of heteroscedasticity (Engle, 1995). The governing equations are

$$
\begin{aligned}
y_{t} & =\varphi_{1} y_{t-1}+\ldots+\varphi_{m} y_{t-m}+\varepsilon_{t} & \varepsilon_{\tau} & =\sigma_{t} \varepsilon_{t} \\
\sigma_{t}^{2} & =\varpi+\alpha \varepsilon_{t-1}^{2}+\beta \sigma_{t-1}^{2} & & e_{t} \approx N(0,1)
\end{aligned}
$$

where $\sigma_{t}$ denotes conditional standard deviation and $e_{t}$ is normal white noise. First, we employed the simplest $\operatorname{GARCH}(1,1)$ model, which was quite efficient and in most cases, only one autoregressive term was statistically significant. The results obtained are presented in the following Table 4 .

Table 4

Parameters of AR-GARCH Models Used

\begin{tabular}{|l|c|c|c|c|c|c|c|c|}
\hline & BUX & PX50 & SKSM & WIG & CAC & DAX & MIBTEL & UKX \\
\hline$\varphi(01)$ & +0.071 & +0.090 & & +0.074 & & & & -0.057 \\
\hline$\varphi(02)$ & & & & & & & & \\
\hline$\varphi(04)$ & & +0.054 & & & & & +0.075 & \\
\hline$\varphi(08)$ & & & +0.084 & & +0.052 & & & \\
\hline$\varphi(10)$ & & & & & & -0.055 & & -0.054 \\
\hline$\omega$ & 0.063 & 0.106 & 0.157 & 0.017 & 0.025 & 0.048 & 0.013 & 0.028 \\
\hline$\alpha$ & 0.053 & 0.091 & 0.056 & 0.045 & 0.056 & 0.076 & 0.072 & 0.093 \\
\hline$\beta$ & 0.926 & 0.853 & 0.872 & 0.950 & 0.935 & 0.911 & 0.925 & 0.890 \\
\hline
\end{tabular}

Clearly, there is strong direct dependence of conditional standard deviation on its previous values, manifested itself by large values of $\beta$ parameter. Second, $\beta$ values vary only slightly among individual stocks returns.

Second, EGARCH $(1,1)$ model was employed to model possible asymmetric reaction with respect to positive and negative shocks $e_{t}$. General form of EGARCH models can be written as (Nelson, 1991):

$$
\log \left(\sigma_{t}^{2}\right)=\varpi+\sum_{j=1}^{p} \beta_{j} \log \left(\sigma_{t-j}^{2}\right)+\sum_{i=1}^{q}\left(\alpha_{i}\left|\frac{X_{t-i}}{\sigma_{t-i}}\right|+\gamma_{i} \frac{X_{t-i}}{\sigma_{t-i}}\right)
$$

Clearly, if $\gamma_{1}=0$, then both positive and negative shocks exert the same influence on volatility. On the other hand, for $-1<\gamma_{1}<0$, a positive shocks influence volatility less 
than a negative ones. Indeed, this second case actually occurs, as can be seen from the Table 5 .

Table 5

Estimated Values of $\gamma_{1}$ Parameter - EGARCH(1,1) Model

\begin{tabular}{|l|c|c|c|c|c|c|c|c|}
\hline & $\boldsymbol{B U X}$ & PX50 & SKSM & WIG & CAC & DAX & MIBTEL & UKX \\
\hline$\gamma_{1}$ & -0.063 & -0.051 & 0.004 & -0.026 & -0.081 & -0.089 & -0.062 & -0.102 \\
\hline$p-$ value & 0.000 & 0.002 & 0.773 & 0.065 & 0.000 & 0.000 & 0.000 & 0.000 \\
\hline
\end{tabular}

\section{Multidimensional Methods}

The main idea of principal component analysis is the use a few linear combinations of original variables to explain the structure of correlation matrix. We have $k$-dimensional random variable $\mathbf{y}=\left(y_{1}, y_{2}, \ldots, y_{k}\right)^{T}$ and $k$-dimensional vector $\mathbf{v}_{i}=\left(v_{i 1}, v_{i 2}, \ldots, v_{i k}\right)^{T}$. Then

$$
z_{i}=\mathbf{v}_{\mathbf{i}}^{T} \mathbf{y}=v_{i 1} y_{1}+v_{i 2} y_{2}+\ldots+v_{i k} y_{k}
$$

is a linear combination of the random vector $\mathbf{y}$. We want to find linear combinations such that $z_{i}, z_{j}$ are uncorrelated for $i \neq j$ and the variance of $z_{i}$ are the largest possible.

First, the correlation matrix among individual returns was computed (see the Table 3). Further, principal component analysis has been performed for three cases. In the first case, only Central-European markets were considered and two principal components were revealed:

$$
\begin{aligned}
& Z_{1}=+0.60 * \text { R_BUX }+0.58 * \text { R_PX50 }+0.55 * \text { R_WIG }+0.05 * \text { R_SKSM } \\
& Z_{2}=-0.06 * \text { R_BUX }-0.09 * \text { R_PX50 }+0.07 * \text { R_WIG }+0.99 * \text { R_SKSM }
\end{aligned}
$$

Thus, the indices BUX, PX50 and WIG have roughly the same weights, whereas SKSM index exhibits strongly individual behaviour. Here symbol R denotes returns.

Second, only West-European markets were considered and the result was:

$$
\mathrm{Z}_{1}=+0.52 * \mathrm{R} \_ \text {CAC }+0.49 * \mathrm{R} \_\mathrm{DAX}+0.49 * \mathrm{R} \_\mathrm{MIBTEL}+0.49 * \mathrm{R} \_\mathrm{UKX}
$$

Clearly, all indices move in coherence in common direction.

Finally, considering all markets, we arrived at three principal components:

$$
\begin{aligned}
& Z_{1}=+0.32 * \mathrm{R} \_ \text {BUX }+0.30 * \mathrm{R} \_\mathrm{PX} 50+0.25 * \mathrm{R} \_\mathrm{WIG}+0.02 * \mathrm{R} \_\mathrm{SKSM} \\
& +0.44 * \mathrm{R} \_\mathrm{CAC}+0.43 * \mathrm{R} \_\mathrm{DAX}+0.43 * \mathrm{R} \_\mathrm{MIBTEL}+0.42 * \mathrm{R} \_\mathrm{UKX} \\
& Z_{2}=-0.60 * \mathrm{R} \_ \text {WIG }-0.42 * \mathrm{R} \_\mathrm{BUX}-0.42 * \mathrm{R} \_\mathrm{PX} 50-0.22 * \mathrm{R} \_\mathrm{SKSM} \\
& +0.28 * \mathrm{R} \_\mathrm{CAC}+0.24 * \mathrm{R} \_\mathrm{UKX}+0.23 * \mathrm{R} \_\mathrm{DAX}+0.23 * \mathrm{R} \_\mathrm{MIBTEL} \\
& Z_{3}=+0.97 * \text { R_SKSM }-0.17 * \text { R_PX50 }-0.14 * \text { R_BUX }-0.05 * \text { R_WIG } \\
& +0.08 * \mathrm{R} \_\mathrm{UKX}+0.05 * \mathrm{R} \_\mathrm{CAC}+0.04 * \mathrm{R} \_\mathrm{DAX}+0.04 * \mathrm{R} \_\mathrm{MIBTEL}
\end{aligned}
$$


Here the first principal component expresses the common part of general trend (apart from SKSM), whereas the second one manifests the discrepancies among Central-European and West-European markets. Once again, the third principal components reflects individual behaviour of SKSM index.

Further, using nearest neighbour clustering method and squared Euclidean metric, we arrived at the following agglomeration schedule:

Figure 4

Agglomeration Schedule: Cluster Analysis of Indices Returns

Dendrogram

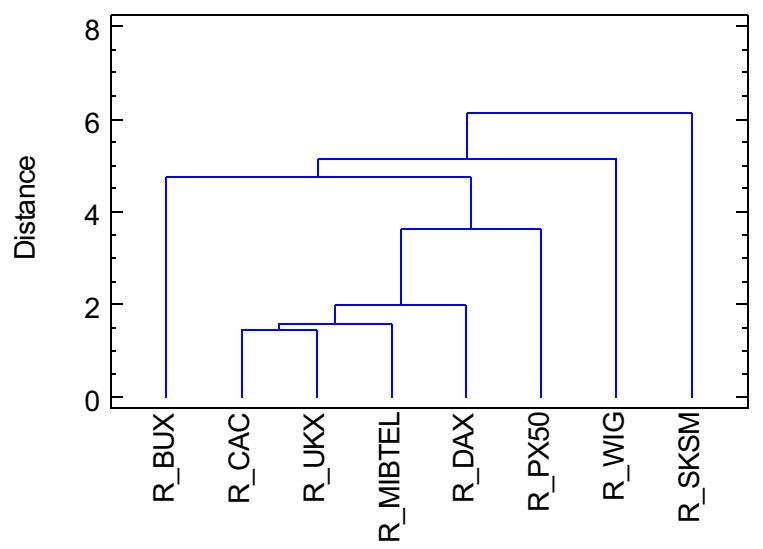

Thus, the agglomeration schedule clearly signalizes the creation of the first cluster consisting of West-European returns CAC $\rightarrow$ UKX $\rightarrow$ MIBTEL $\rightarrow$ DAX. As for emerging markets, the sequence of clustering is PX50 $\rightarrow$ BUX $\rightarrow$ WIG $\rightarrow$ SKSM. Once again, the behaviour of Slovak SKSM index is proved to be anomalous.

\section{Vector Autoregression Models (VAR)}

For deeper understanding of dynamical dependencies among the returns of different stock indices, vector autoregression models were introduced. Multivariate time series represents a vector of $n$ different time series observed simultaneously. In principle, time series with the same basic characteristics can be analysed, i.e. individual stochastic processes creating $\mathbf{y}_{t}$ vector should be either stationary or integrated of the same order. As known, a vector autoregression model ( VAR) is a generalization of one-dimensional AR model (Hamilton, 1994). Clearly, the simplest form creates VAR(1) model for bivariate system which can be written as

$$
\begin{aligned}
& y_{1, t}=\alpha_{10}+\alpha_{11} y_{1, t-1}+\alpha_{12} y_{2, t-1}+u_{1, t} \\
& y_{2, t}=\alpha_{20}+\alpha_{21} y_{1, t-1}+\alpha_{22} y_{2, t-1}+u_{2, t}
\end{aligned}
$$

or in matrix form 


$$
\mathbf{y}_{t}=\alpha_{0}+\mathbf{A y}_{t-1}+\mathbf{u}_{t} \quad \mathbf{A}=\left[\begin{array}{ll}
\alpha_{11} & \alpha_{12} \\
\alpha_{21} & \alpha_{22}
\end{array}\right]
$$

General $p$-dimensional model $\operatorname{VAR}(\mathrm{p})$ has the form

$$
\mathbf{y}_{t}=\alpha_{0}+\mathbf{A}_{1} \mathbf{y}_{t-1}+\mathbf{A}_{2} \mathbf{y}_{t-2}+\ldots+\mathbf{u}_{t}
$$

Further, it is known, an one-dimensional AR(p) process is stationary only if the roots of characteristic equation

$$
1-\alpha_{1} x^{1}+\alpha_{2} x^{2}-\ldots-\alpha_{p} x^{p}=0
$$

lie outside unit circle. Similarly, in the case of multidimensional VAR(p) process, characteristic equation can be written as

$$
\left|\mathbf{I}-\mathbf{A}_{\mathbf{1}} z^{1}-\mathbf{A}_{\mathbf{2}} z^{2}-\ldots-\mathbf{A}_{p} z^{p}\right|=0
$$

Finally, for bivariate VAR(1) process, this condition is reduced to the solution of

$$
1-\operatorname{tr}(\mathbf{A}) z+|\mathbf{A}| z^{2}=0
$$

To investigate dynamical dependence among individual returns, Granger causality test was applied in two-dimensional system of jointly stationary time series (Alexander, 2001). We say, variable $x$ Granger cause variable $y$, if delayed values of $x$ variable improve prediction of $y$, despite that delayed values of $y$ are introduced as explanatory variables. The model assumed is bivariate $\operatorname{VAR}(\mathrm{p})$ in the form

$$
\begin{aligned}
& x_{t}=c_{1}+\sum_{i=1}^{p} \alpha_{1 i} x_{t-i}+\sum_{i=1}^{p} \beta_{1 i} y_{t-i}+u_{1 t} \\
& y_{t}=c_{2}+\sum_{i=1}^{p} \alpha_{2 i} x_{t-i}+\sum_{i=1}^{p} \beta_{2 i} y_{t-i}+u_{1 t}
\end{aligned}
$$

Then the test of Granger causality in direction $x \rightarrow y$ can be understood as $F$-test of parameters $\alpha_{21}, \alpha_{22}, \ldots, \alpha_{2 p}$ in regression model (5.7), whereas the test of Granger causality in direction $x \rightarrow y$ is related to parameters $\beta_{11}, \beta_{12}, \ldots, \beta_{1 p}$. The results obtained are summarized in the Tables $6,7,8$.

Table 6

Granger Causality Test: P-values for the Returns between Central-European Indices and Time Lags 1, 2, 3, 4, 5

\begin{tabular}{|l|l|l|l|l|l|}
\hline $\mathrm{SKSM} \rightarrow \mathrm{BUX}$ & 0.489 & $\mathrm{SKSM} \rightarrow$ WIG & 0.815 & $\mathrm{SKSM} \rightarrow$ PX50 & 0.564 \\
\hline $\mathrm{BUX} \rightarrow$ SKSM & 0.200 & WIG $\rightarrow$ SKSM & 0.132 & PX50 $\rightarrow$ SKSM & 0.073 \\
\hline BUX $\rightarrow$ WIG & $\mathbf{0 . 0 0 0}$ & BUX $\rightarrow$ PX50 & 0.169 & WIG $\rightarrow$ PX50 & 0.181 \\
\hline WIG $\rightarrow$ BUX & $\mathbf{0 . 0 0 6}$ & PX50 $\rightarrow$ BUX & $\mathbf{0 . 0 3 1}$ & PX50 $\rightarrow$ WIG & $\mathbf{0 . 0 0 2}$ \\
\hline
\end{tabular}


Table 7

Granger Causality Test: P-values for the Returns between West-European Indices and Time Lags 1, 2, 3, 4, 5

\begin{tabular}{|l|l|l|l|l|l|}
\hline $\mathrm{MIBTEL} \rightarrow$ UKX & 0.202 & MIBTEL $\rightarrow$ CAC & $\mathbf{0 . 0 0 0}$ & MIBTEL $\rightarrow$ DAX & $\mathbf{0 . 0 1 0}$ \\
\hline $\mathrm{UKX} \rightarrow$ MIBTEL & 0.067 & CAC $\rightarrow$ MIBTEL & 0.534 & DAX $\rightarrow$ MIBTEL & 0.078 \\
\hline $\mathbf{U K X} \rightarrow$ CAC & $\mathbf{0 . 0 0 4}$ & UKX $\rightarrow$ DAX & $\mathbf{0 . 0 0 1}$ & CAC $\rightarrow$ DAX & $\mathbf{0 . 0 1 4}$ \\
\hline $\mathrm{CAC} \rightarrow$ UKX & 0.158 & DAX $\rightarrow$ UKX & $\mathbf{0 . 0 0 0}$ & DAX $\rightarrow$ CAC & $\mathbf{0 . 0 0 0}$ \\
\hline
\end{tabular}

Table 8

Granger Causality Test: P-values for the Returns between Central- and West- European Indices and Time Lags 1, 2, 3, 4, 5

\begin{tabular}{|l|l|l|l|l|l|l|l|}
\hline SKSM $\rightarrow$ MIBTEL & 0.918 & SKSM $\rightarrow$ UKX & 0.505 & SKSM $\rightarrow$ CAC & 0.860 & SKSM $\rightarrow$ DAX & 0.770 \\
\hline MIBTEL $\rightarrow$ SKSM & $\mathbf{0 . 0 0 4}$ & UKX $\rightarrow$ SKSM & 0.211 & CAC $\rightarrow$ SKSM & $\mathbf{0 . 0 2 2}$ & DAX $\rightarrow$ SKSM & $\mathbf{0 . 0 1 2}$ \\
\hline BUX $\rightarrow$ MIBTEL & 0.576 & BUX $\rightarrow$ UKX & 0.583 & BUX $\rightarrow$ CAC & 0.562 & BUX $\rightarrow$ DAX & 0.314 \\
\hline MIBTEL $\rightarrow$ BUX & $\mathbf{0 . 0 4 5}$ & UKX $\rightarrow$ BUX & $\mathbf{0 . 0 0 1}$ & CAC $\rightarrow$ BUX & 0.081 & DAX $\rightarrow$ BUX & 0.054 \\
\hline WIG $\rightarrow$ MIBTEL & 0.060 & WIG $\rightarrow$ UKX & 0.325 & WIG $\rightarrow$ CAC & $\mathbf{0 . 0 0 8}$ & WIG $\rightarrow$ DAX & $\mathbf{0 . 0 0 4}$ \\
\hline MIBTEL $\rightarrow$ WIG & $\mathbf{0 . 0 0 0}$ & UKX $\rightarrow$ WIG & $\mathbf{0 . 0 0 0}$ & CAC $\rightarrow$ WIG & $\mathbf{0 . 0 0 0}$ & DAX $\rightarrow$ WIG & $\mathbf{0 . 0 0 0}$ \\
\hline PX50 $\rightarrow$ MIBTEL & 0.593 & PX50 $\rightarrow$ UKX & 0.960 & PX50 $\rightarrow$ CAC & 0.900 & PX $50 \rightarrow$ DAX & 0.494 \\
\hline MIBTEL $\rightarrow$ PX50 & 0.473 & UKX $\rightarrow$ PX50 & 0.191 & CAC $\rightarrow$ PX50 & 0.709 & DAX $\rightarrow$ PX50 & 0.490 \\
\hline
\end{tabular}

Further, it is necessary to take into account the influence of possible cointegration of time series of stock indices. A system of time series $x, y \sim I(1)$ is cointegrated, if such $\alpha$ exists, then a linear combination $z=x-\alpha y \sim I(0)$. Thus, a cointegration vector $(1,-\alpha)$ contains the weights in $z$. For cointegration testing, Johansen test is usually used (Hamilton, 1994). It is based on the eigenvalues of a stochastic matrix and reduces to a canonical correlation problem similar to that of principal components. It is a multivariate generalization of the unit root test. $\operatorname{VAR}(p)$ model (5.3) can be easily rewritten into the form

$\Delta \mathbf{y}_{t}=\alpha_{0}+\left(\mathbf{A}_{1}-\mathbf{I}\right) \mathbf{y}_{t-1}+\left(\mathbf{A}_{1}+\mathbf{A}_{2}-\mathbf{I}\right) \mathbf{y}_{t-2}+\ldots+\left(\mathbf{A}_{1}+\mathbf{A}_{2}+\ldots+\mathbf{A}_{p}-\mathbf{I}\right) \mathbf{y}_{t-p}+\mathbf{u}_{t}$

and Johansen test becomes a test for the number of non-zero eigenvalues of the matrix

$$
\Pi=\mathbf{A}_{1}+\mathbf{A}_{2}+\ldots+\mathbf{A}_{p}-\mathbf{I}
$$

Johansen and Juselius recommended the use of the standard test of the trace of matrix for the number $r$ of its non-zero eigenvalues

$$
H_{0}: r \leq R \quad H_{1}: r>R \quad T=-n \sum_{i=R+1}^{k} \ln \left(1-\lambda_{i}\right)
$$

where $T$ denotes test statistic, $n$ sample size, $k$ numer of variables and $\lambda_{i} i$-th eigenvalue according to magnitude. In the case of two cointegrated time series $x, y$, dynamical Error Correction Model (ECM) can be used for the first differences of integrated variables I(1). Then it holds (Hamilton, 1994) 


$$
\begin{aligned}
& \Delta x_{t}=\alpha_{1}+\sum_{i} \beta_{1 i} \Delta x_{t-i}+\sum_{i} \beta_{2 i} \Delta x_{t-i}+\gamma_{1} z_{t-1}+u_{1 t} \\
& \Delta y_{t}=\alpha_{2}+\sum_{i} \beta_{3 i} \Delta x_{t-i}+\sum_{i} \beta_{4 i} \Delta x_{t-i}+\gamma_{2} z_{t-1}+u_{2 t}
\end{aligned}
$$

The results obtained are compiled in the following tables.

\section{Table 9}

Returns of Central-European Indices:

P-values of Trace Tests, Cointegration Vectors and Coefficients in ECM Model $(i=1,2,3,4,5)$

\begin{tabular}{|l|c|c|c|c|c|c|c|}
\hline & Trace & $\mathbf{z}$ & $\mathbf{1}$ & $\mathbf{2}$ & $\mathbf{3}$ & $\mathbf{4}$ & $\mathbf{5}$ \\
\hline $\mathrm{SKSM} \rightarrow \mathrm{BUX}$ & 0.112 & - & - & - & - & - & - \\
\hline $\mathrm{BUX} \rightarrow \mathrm{SKSM}$ & 0.112 & - & - & - & - & - & - \\
\hline $\mathrm{SKSM} \rightarrow$ WIG & 0.316 & - & - & - & - & - & - \\
\hline $\mathrm{WIG} \rightarrow \mathrm{SKSM}$ & 0.316 & - & - & +0.055 & - & - & - \\
\hline $\mathrm{SKSM} \rightarrow \mathrm{PX} 50$ & 0.137 & - & - & - & - & - & - \\
\hline $\mathrm{PX} 50 \rightarrow$ SKSM & 0.137 & - & - & - & - & - & -0.066 \\
\hline $\mathrm{WIG} \rightarrow$ BUX & 0.089 & - & +0.095 & - & - & - & - \\
\hline $\mathrm{BUX} \rightarrow$ WIG & 0.089 & - & +0.136 & - & - & +0.061 & - \\
\hline $\mathrm{WIG} \rightarrow \mathrm{PX} 50$ & 0.024 & $(1,-0.753)$ & +0.050 & - & - & - & - \\
\hline $\mathrm{PX} 50 \rightarrow$ WIG & 0.024 & & +0.102 & - & - & +0.092 & - \\
\hline $\mathrm{BUX} \rightarrow \mathrm{PX50}$ & 0.242 & - & - & - & - & - & - \\
\hline $\mathrm{PX} 50 \rightarrow$ BUX & 0.242 & - & - & - & - & +0.110 & - \\
\hline
\end{tabular}

There is statistically significant cointegration between PX50 and WIG indices and only slightly nonsignificant one between BUX and WIG $(p=0.089)$. The strongest influence from preceeding returns of BUX to WIG amounts to 0.136 and, further, from PX50 to WIG. Thus, Poland capital market seems to be influenced both by the Czech Republic and Hungary. The same is true also for the influence of returns delayed by four days.

Table 10

Returns of West-European Indices:

P-values of Trace Tests, Cointegration Vectors and Coefficients in ECM Model $(i=1,2,3,4,5)$

\begin{tabular}{|l|c|c|c|c|c|c|c|}
\hline & Trace & $\mathbf{z}$ & $\mathbf{1}$ & $\mathbf{2}$ & $\mathbf{3}$ & $\mathbf{4}$ & $\mathbf{5}$ \\
\hline $\mathrm{MIBTEL} \rightarrow \mathrm{UKX}$ & 0.174 & - & +0.066 & - & - & - & - \\
\hline $\mathrm{UKX} \rightarrow \mathrm{MIBTEL}$ & 0.174 & - & +0.078 & - & - & - & -0.090 \\
\hline $\mathrm{MIBTEL} \rightarrow \mathrm{CAC}$ & 0.064 & - & +0.150 & - & - & +0.109 & - \\
\hline $\mathrm{CAC} \rightarrow \mathrm{MIBTEL}$ & 0.064 & - & - & - & - & - & - \\
\hline $\mathrm{MIBTEL} \rightarrow \mathrm{DAX}$ & 0.201 & - & +0.146 & - & - & +0.111 & - \\
\hline $\mathrm{DAX} \rightarrow \mathrm{MIBTEL}$ & 0.201 & - & +0.081 & - & - & - & - \\
\hline $\mathrm{UKX} \rightarrow \mathrm{CAC}$ & 0.023 & $(1,-0.649)$ & - & - & - & +0.138 & -0.115 \\
\hline $\mathrm{CAC} \rightarrow \mathrm{UKX}$ & 0.023 & & - & - & - & - & - \\
\hline $\mathrm{UKX} \rightarrow$ DAX & 0.038 & $(1,-0.652)$ & +0.117 & - & - & +0.116 & -0.147 \\
\hline $\mathrm{DAX} \rightarrow \mathrm{UKX}$ & 0.038 & & +0.103 & - & - & - & - \\
\hline $\mathrm{CAC} \rightarrow \mathrm{DAX}$ & 0.125 & - & +0.121 & +0.128 & - & - & - \\
\hline $\mathrm{DAX} \rightarrow \mathrm{CAC}$ & 0.125 & - & +0.205 & - & - & - & - \\
\hline
\end{tabular}


In the case of West-European markets, statistically significant cointegration was found between CAC and UKX and between DAX and UKX price series. Influences from preceeding returns are generally stronger than in the case of Central European time series. Second, the influences of UKX and MIBTEL seem to control the development of DAX and CAC. The same conclusion holds for the influence of returns delayed by 4 and 5 .

Table 11

Returns of Central-European and West-European Indices:

P-values of Trace Tests, Cointegration Vectors and Coefficients in ECM Model $(i=1,2,3,4,5)$

\begin{tabular}{|c|c|c|c|c|c|c|c|}
\hline & Trace & $\mathbf{z}$ & 1 & 2 & 3 & 4 & 5 \\
\hline $\mathrm{SKSM} \rightarrow \mathrm{MIBTEL}$ & 0.522 & - & - & - & - & - & - \\
\hline MIBTEL $\rightarrow$ SKSM & & & - & - & - & - & -0.101 \\
\hline $\mathrm{SKSM} \rightarrow \mathrm{UKX}$ & 0.648 & - & - & - & - & - & - \\
\hline $\mathrm{UKX} \rightarrow \mathrm{SKSM}$ & & & - & - & - & - & -0.065 \\
\hline $\mathrm{SKSM} \rightarrow \mathrm{CAC}$ & 0.471 & - & - & - & - & - & - \\
\hline $\mathrm{CAC} \rightarrow \mathrm{SKSM}$ & & & - & - & - & - & -0.073 \\
\hline $\mathrm{SKSM} \rightarrow \mathrm{DAX}$ & 0.844 & - & - & - & - & - & - \\
\hline $\mathrm{DAX} \rightarrow \mathrm{SKSM}$ & & & - & - & - & - & -0.072 \\
\hline BUX $\rightarrow$ MIBTEL & 0.633 & - & - & - & - & - & - \\
\hline MIBTEL $\rightarrow$ BUX & & & +0.099 & - & - & - & - \\
\hline $\mathrm{BUX} \rightarrow \mathrm{UKX}$ & 0.943 & - & - & - & - & - & - \\
\hline $\mathrm{UKX} \rightarrow \mathrm{BUX}$ & & & +0.148 & - & - & - & - \\
\hline $\mathrm{BUX} \rightarrow \mathrm{CAC}$ & 0.890 & - & - & - & - & - & - \\
\hline $\mathrm{CAC} \rightarrow \mathrm{BUX}$ & & & +0.072 & - & - & +0.061 & - \\
\hline $\mathrm{BUX} \rightarrow \mathrm{DAX}$ & 0.799 & - & - & - & - & - & +0.054 \\
\hline $\mathrm{DAX} \rightarrow \mathrm{BUX}$ & & & +0.074 & - & - & - & - \\
\hline WIG $\rightarrow$ MIBTEL & 0.396 & - & +0.052 & - & - & - & - \\
\hline MIBTEL $\rightarrow$ WIG & & & +0.208 & - & - & - & - \\
\hline $\mathrm{WIG} \rightarrow \mathrm{UKX}$ & 0.954 & - & - & - & - & - & - \\
\hline $\mathrm{UKX} \rightarrow \mathrm{WIG}$ & & & +0.221 & - & - & +0.092 & - \\
\hline $\mathrm{WIG} \rightarrow \mathrm{CAC}$ & 0.583 & - & +0.083 & - & - & - & - \\
\hline $\mathrm{CAC} \rightarrow \mathrm{WIG}$ & & & +0.168 & - & - & +0.055 & - \\
\hline $\mathrm{WIG} \rightarrow \mathrm{DAX}$ & 0.470 & - & +0.097 & - & - & - & - \\
\hline $\mathrm{DAX} \rightarrow \mathrm{WIG}$ & & & +0.143 & - & - & +0.055 & +0.074 \\
\hline PX50 $\rightarrow$ MIBTEL & 0.641 & - & - & - & - & - & - \\
\hline MIBTEL $\rightarrow$ PX50 & & & - & - & - & - & - \\
\hline $\mathrm{PX} 50 \rightarrow \mathrm{UKX}$ & 0.920 & - & - & - & - & - & - \\
\hline $\mathrm{UKX} \rightarrow \mathrm{PX} 50$ & & & - & - & - & - & - \\
\hline $\mathrm{PX} 50 \rightarrow \mathrm{CAC}$ & 0.784 & - & - & - & - & - & - \\
\hline $\mathrm{CAC} \rightarrow \mathrm{PX} 50$ & & & - & - & - & - & - \\
\hline $\mathrm{PX} 50 \rightarrow \mathrm{DAX}$ & 0.697 & - & - & - & - & - & - \\
\hline $\mathrm{DAX} \rightarrow \mathrm{PX} 50$ & & & - & - & - & - & - \\
\hline
\end{tabular}

The results obtained indicate that BUX and WIG behaviour is influenced by all West-European capital markets. On the other hand, no influence was revealed in the case of PX50 index, whereas SKSM index is influenced in reverse sense. 


\section{Conclusion}

As for returns summary statistics, the kurtosis of empirical distribution was always positive and, therefore, logistic or Student's t distributions proved to be always better fitting in comparison with the normal one; the half of values occurs roughly within $\pm 1 \%$. Further, unconditional standard deviation seems to be very quiet; on the other hand, one can expect sudden onsets of increased volatility during some periods.

The highest correlations occur on the one hand among West-European indices CAC, UKX, DAX, MIBTEL; on the other hand, as for Central-European indices, the correlations are weaker and the highest ones occur among BUX, PX-50 and WIG, whereas SKSM returns exhibit some kind of nontypical behaviour. The same conclusions were drawn with the use of cluster analysis and principal component analysis. The structure of autocorrelation function (ACF) exhibits several typical features. On the one hand, ACF values are rather small, but, on the other hand, they are persistent up to relatively high order.

As for modelling, the combination of an autoregressive model and GARCH $(1,1)$ model was sufficient to remove present heteroscedasticity. In all cases, there was strong dependence of conditional variance on its previous value. Thus, the ARMA models are not capable to catch features observed and there is strong need for GARCH models. In addition, modelling with EGARCH $(1,1)$ models revealed that positive shocks influence volatility less than a negative ones.

Both Granger causality tests and VEC models signalize strong influence of West-European markets on Central-European ones. The strongest influence comes from preceeding returns at time $t-1$, but returns with lags 4,5 are also significant in several cases. As for Central-European markets, the influence of the Czech and Hungary markets proved to be dominant.

\section{References}

Alexander, C. (2001), "Market Models." Wiley.

Cipra, T. (1986), "Analýza časových řad s aplikacemi v ekonomii."Praha : SNTL.

Engle, R. (1995), "ARCH (Selected Readings). "Oxford : Oxford University Press.

Evans, M., Hastings, N., Peacock, B. (1993), "Statistical Distributions."Wiley.

Fama, E.F. (1968), "Risk, Return and Equilibrium: Some Clarifying Comments." Journal of Finance, 23 (1), pp. 29-40.

Hamilton, J.D. (1994), "Time Series Analysis.” Princeton : Princeton University Press.

Hull, J.C. (2006), "Options, Futures and Other Derivatives." Prentice Hall.

Johnson, N., Kotz, S., Balakrishnan, N. (1995), "Continuous Univariate Distributions." Wiley.

Markowitz, H. (1952), "Portfolio Selection." Journal of Finance, 7 (1), pp. 77-91.

Mossin, J. (1966), "Equilibrium in a Capital Asset Market." Econometrica, 34 (4), pp. 768-783.

Nelson, D.B. (1991), "Conditional Heteroskedasicity in Asset Returns: A New Approach." Econometrica. 59, pp. 347-370.

Sharpe, W.F. (1964), "Capital Asset Prices: A Theory of Market Equilibrium under Conditions of Risk." Journal of Finance, 19 (3), pp. 425-442.

Sharpe, W.F., Alexander, G.J. (1990), “Investments.” Prentice Hall.

Solnik, B. (1996), "International Investments." Addison-Wesley.

Tsay, R.S. (2002), "Analysis of Financial Time Series. "Wiley. 